\title{
Formation, Evolution, and the Structure of Fronts Produced By Unsteady Injection of Highly Magnetized, Relativistic Flows
}

\author{
Amir Levinson * \\ CRSR, Cornell University, Ithaca, NY 14853 \\ and \\ Maurice H.P.M. van Putten \\ Math Department, MIT, Cambridge, MA 02139
}

\begin{abstract}
We study the formation, evolution and structure of dissipative fronts produced by overtaking collisions of relativistic streams, with emphasis on strongly magnetized flows. The evolution of the system is followed using analytical approach in the simple wave regime and numerical simulations in the non-simple wave regime, until a steady-state is reached. The steady-state structure of the front is then examined by solving the appropriate jump conditions. The conversion of magnetic energy into kinetic energy is parametrized in terms of the Alfvén 4-velocity inside the front. The implications for gammaray jets are briefly discussed.

Keywords : galaxies:jets-MHD-relativity-shock waves
\end{abstract}

\section{INTRODUCTION}

Highly magnetized, relativistic outflows are believed to play an important role in a variety of astrophysical systems, e.g., pulsar winds (Kennel \& Coroniti 1984; Gallant, et al. 1992), Galactic and extragalactic superluminal sources (Burns \& Lovelace 1982; Begelman \& Li, 1994; Levinson \& Blandford 1996), gamma-ray bursts (Usov 1992; Waxman 1995; Thompson \& Duncan 1993; Smolsky \& Usov 1996), and others. Such flows are likely to be unsteady,

\footnotetext{
*Present address: School of Physics and Astronomy, Tel Aviv University, Tel Aviv 69978, Israel
} 
giving rise to formation of shocks which can lead ultimately to the dissipation of some fraction of the bulk energy, either in the form of thermal energy or, through shock acceleration (Blandford \& Eichler 1987) or magnetic reconnection, high energy particles with nonthermal distributions. As demonstrated by Romanova \& Lovelace (1997) recently, the creation of dissipative fronts in Poynting flux jets may give rise to $\gamma$-ray flares as often seen in blazars.

One important difference between unmagnetized and highly magnetized flows is that, in the former case the sound speed is limited to $c / \sqrt{3}$, whereas in the latter case the Lorentz factor associated with the speed of propagation of a disturbance - the magnetosonic speed can be much larger. Consequently, the conditions required for the formation of strong shocks are expected to be more stringent in highly magnetized flows. Even when the upstream flow is supermagnetosonic, so that strong shocks are created, only a relatively small fraction of the incoming flow energy goes to heat the downstream region; the remainder is used up to slightly compress the magnetic field downstream (Kennel \& Coroniti 1984). The situation might be markedly different however in the presence of rapid magnetic field dissipation behind the shock.

Motivated by the above considerations, we study in this paper the formation, evolution, and structure of fronts produced by unsteady injection of relativistic streams, with particular emphasis on flows in which the energy flux is dominated by electromagnetic fields (commonly referred to as high sigma flows).

\section{THEORY}

\section{A. Basic equations}

The stress-energy tensor of a perfectly conducting fluid can be written in terms of $u^{\alpha}$, the fluid 4-velocity, and the magnetic field 4-vector $b^{\alpha}=\tilde{F}^{\alpha \beta} u_{\beta}$, where $\tilde{F}^{\alpha \beta}$ is the dual electromagnetic stress-tensor, as

$$
T^{\alpha \beta}=r h^{*} u^{\alpha} u^{\beta}+p^{*} g^{\alpha \beta}-b^{\alpha} b^{\beta} .
$$

Here, $g_{\alpha \beta}$ denotes the metric tensor, $r$ is the fluid rest mass density, $h^{*}=h+b^{2} / r$ and $p^{*}=p+b^{2} / 2$, where $p$ is the proper gas pressure, $h$ the specific enthalpy and $b^{2}=b^{\alpha} b_{\alpha}$. Under the assumption of ideal MHD, Ohm's law reduces to the condition $F^{\alpha \beta} u^{\beta}=0$. The MHD equations can then be written in a divergence form as (van Putten, 1993)

$$
\begin{aligned}
\nabla_{\alpha} T^{\alpha \beta} & =0, \\
\nabla_{\alpha}\left(r u^{\alpha}\right) & =0, \\
\nabla_{\alpha}\left(u^{[\alpha} b^{\beta]}+g^{\alpha \beta} c\right) & =0,
\end{aligned}
$$

where $c:=b_{\alpha} u^{\alpha}=0$ is a conserved constraint. Note that $\sqrt{4 \pi} b_{\alpha}$ represents now the magnetic field measured in the fluid rest frame, the absolute value of which we denote here by $B$. The 
above set of equations must be supplemented by an equation of state for the gas. In regions of adiabatic flow where $u^{\alpha} \nabla_{\alpha} S=0, S$ being the entropy, the specific enthalpy is given by $h=1+\int d p / r$, with $p=p(r)$.

Using eq. (1), the energy flux carried by the flow can be written in the form

$$
\mathcal{F}=r h \gamma^{2} \mathbf{v}\left(1+u_{A}^{2}\right)=r h \gamma_{A}^{2} \gamma^{2} \mathbf{v}
$$

where $\mathbf{v}$ is the fluid 3-velocity, $u_{A}^{\alpha}=b^{\alpha} / \sqrt{r h}$ is the Alfven 4-velocity with respect to the fluid rest frame, and $\gamma_{A}$ is the corresponding Lorentz factor.

\section{B. Simple waves}

To simplify the analysis, we shall consider in the following $1 \mathrm{D}$ flow with velocity $u^{\alpha}=$ $(\gamma, u, 0,0)$, a polytropic equation of state, $p(r)=K r^{\Gamma}$, and magnetic field perpendicular to the fluid 3-velocity. Using Maxwell equations together with the continuity equation it is readily shown that $B / r$ is conserved along stream lines (de Hoffmann \& Teller 1958). Consequently, the proper magnetic pressure can be expressed as, $p_{B}=\kappa r^{2}$, with $\kappa=$ $B^{2} / 8 \pi r^{2}$. Thus, the problem reduces essentially to that of a $1 \mathrm{D}$ hydrodynamic flow with an effective equation of state $p^{*}(r)=K r^{\Gamma}+\kappa r^{2}$. Next, we define

$$
c_{s}^{2}=\frac{1}{h^{*}} \frac{\partial p^{*}}{\partial r}=\frac{\Gamma K r^{\Gamma-1}+2 \kappa r}{1+\frac{\Gamma}{\Gamma-1} K r^{\Gamma-1}+2 \kappa r} .
$$

Evidently, in the absence of magnetic fields, viz., $\kappa=0, c_{s}$ is simply the sound speed. In the presence of very strong fields, such that the gas pressure is much smaller than the magnetic field pressure, $c_{s}$ reduces to the Alfven speed $u_{A} / \gamma_{A}$. If the entropy and $\kappa$ are taken to be constant throughout the flow, one can define the variables $d \sigma=c_{s} d r / r$ and $\lambda=\sinh ^{-1}(u)$. Equations (2) then yield (van Putten 1991)

$$
\frac{\partial \chi_{ \pm}}{\partial t} \pm A_{ \pm}(\sigma, \lambda) \frac{\partial \chi_{ \pm}}{\partial x}=0
$$

with $\chi_{ \pm}=\sigma \pm \lambda$, and $A_{ \pm}(\sigma, \lambda)=\left(c_{s} \pm v\right) /\left(1 \pm c_{s} v\right)$, where $v=u / \gamma$ is the fluid 3-velocity. Equation (5) implies that $\chi_{+}$and $\chi_{-}$are constant along the characteristics $d x / d t=A_{+}$and $d x / d t=A_{-}$, respectively. Note that $A_{ \pm}$is the relativistic sum and difference of $c_{s}$ and $v$. In the case of simple waves one of the Riemann invariants is a constant. Let us suppose that $\chi_{-}=$const. Then, $A_{+}$depends only on the remaining invariant, $\chi_{+}$, and it is clear from eq. (5) that $\chi_{+}$is constant along straight lines with slopes $A_{+}$, implying that $A_{+}$is simply the speed of propagation of a disturbance in the observer frame. For weak disturbances for which $v \rightarrow 0$, we obtain $A_{+} \rightarrow c_{s}$, verifying that $c_{s}$ is indeed the speed of propagation of the disturbance in the fluid frame.

For illustration, we consider unsteady injection of 1D, highly magnetized fluid with the following initial and boundary conditions: 


$$
\begin{array}{r}
\lambda(x, t)=\lambda_{o}=\text { const } ; \quad t \leq 0, \\
\lambda(x=0, t)=\lambda_{b}(t)=\lambda_{o}+t / \tau ; \quad t>0 .
\end{array}
$$

The general solution is then given by

$$
\lambda(x, t)=\lambda_{b}\left(t^{\prime}\right) ; \quad t^{\prime}=t-\frac{x}{A\left[\lambda_{b}\left(t^{\prime}\right)\right]},
$$

where the subscript $(+)$ has been dropped to simplify the notation. In order to solve for $t^{\prime}$, we must determine $A(\lambda)$. Since we are merely interested in high sigma flows, we can, to a good approximation, neglect the gas pressure. This corresponds to the limit $K=0$ in equation (4). We then obtain $A(\lambda)=\tanh \left(3 \lambda / 2-\lambda_{o} / 2+\lambda_{A o}\right)$, where $\sinh \left(\lambda_{A o}\right)=u_{A o}$ is the Alfv́en 4 -velocity of the initial flow (at $t \leq 0$ ). On substituting the later expression for $A(\lambda)$ into eq. (7), we obtain a transcendental equation for the retarded time $t^{\prime}$, which can be solved numerically. The solution thereby obtained describes a steepening wave propagating at a velocity $\tanh \left(\lambda_{o}+\lambda_{A o}\right)$, the relativistic sum of $v_{A o}$ and $v$. This wave will steepen into a shock after time $t_{s}$ at some distance $x_{s}$ from the injection point. The point $\left(t_{s}, x_{s}\right)$ is a pole of the function $\lambda(x, t)$, and can be found from the equation $d \lambda / d x=\infty$. The result is

$$
t_{s}=\frac{\tau}{3} \sinh \left(2 \lambda_{o}+2 \lambda_{A o}\right) ; \quad x_{s}=c t_{s} \tanh \left(\lambda_{o}+\lambda_{A o}\right)
$$

If we associate the flow acceleration time $\tau$ with the size of the injection region, $R$, (this might be, for example, the radius of a black hole or the inner boundary of an accretion disk from which a jet is ejected), then in the ultra-relativistic limit eq. (8) gives, $x_{s}=R \gamma_{o}^{2} \gamma_{A}^{2} / 3$. Note that in this limit $\gamma_{o} \gamma_{A}$ is just the Lorentz factor associated with the speed of propagation of the disturbance with respect to the injection frame, so that $c \tau\left(\gamma_{o} \gamma_{A}\right)^{2}$ is roughly the distance from the injection point at which two disturbances separated by a time $\tau$ collide. This constraint on the location at which the front is created may have important implications for the characteristics of the high-energy emission from relativistic jets (Levinson 1996). In the case of the Crab pulsar the above criterion (although the different geometry is expected to somewhat alter this result) implies that for $R \sim 10^{6} \mathrm{~cm}$ any fluctuations of the pulsar wind are not likely to steepen into traveling shocks upstream the standing shock (which is produced as a result of the interaction of the pulsar wind with the confining, slowly expanding SNR, and is located at a radius of about $0.1 \mathrm{pc})$, unless $\gamma_{o} \gamma_{A}<10^{5}$.

\section{The evolution and structure of adiabatic fronts}

The simple wave solution breaks down after a shock is formed (i.e., for $t>t_{s}$ ). To study the evolution of the front in the non-simple wave regime, we have solved equations (2) numerically. A detailed account of the numerical method used is given in van Putten (1993). To test the code, we have compared the numerical results with the analytic solution obtained above in the simple wave regime. The agreement is found to be good. 
An example is shown in fig. 1. As seen from this example, the collision of the initial and injected fluids leads ultimately to the creation of a front consisting of two shocks moving away from each other, and a contact discontinuity across which the total pressure is continuous. The front propagates at a speed (i.e., the speed of the contact discontinuity) intermediate between that of the initial and injected flows, and expands at a rate proportional to the relative velocity of the two shocks. Consequently, there is a net energy flow into the front, giving rise to a pressure buildup inside. After the injection is terminated, the front reaches a steady-state whereby the net energy flow into the front is balanced by adiabatic cooling, owing to the expansion of the front. It is then convenient to explore the steady-state structure of the front using the appropriate jump conditions. Now, in the ideal MHD limit the specific magnetic field, $B / r$, must be continuous across the shock. This will no longer be true in the presence of magnetic field dissipation, which may result from e.g., magnetic reconnection in the front, (this would require magnetic field topology more complicated than that invoked above). A self consistent treatment of dissipative fronts is beyond the scope of this Letter. In order to explore the effect of magnetic field dissipation on the parameters of the shocked fluid, we suppose that some fraction of the magnetic field energy is being converted into particle energy inside the front by some unspecified mechanism. We can then treat the magnetic field behind each shock as a free parameter which lies in the range between zero (although the magnetic field is not likely to drop well below the equipartition value) and the maximum value obtained in the ideal MHD limit.

The problem can be most easily solved in the rest frame of the front. In this frame the jump conditions decouple and reduce essentially to those of two single, independent shocks. Let subscript $-(+)$ refers to fluid quantities leftward (rightward) of the contact discontinuity, and denote quantities inside the front by subscript $f$. The jump conditions at the right $(+)$ and left (-) shocks can then be written in the front frame as,

$$
\begin{aligned}
& r_{f \pm}=\gamma_{ \pm}\left(1-v_{ \pm} / v_{s \pm}\right) r_{ \pm} \\
& r_{ \pm} h_{ \pm}^{*} \gamma_{ \pm}^{2}\left(1-v_{ \pm} / v_{s \pm}\right)-p_{ \pm}^{*}=r_{f \pm} h_{f \pm}^{*}-p_{f \pm}^{*} \\
& r_{ \pm} h_{ \pm}^{*} \gamma_{ \pm}^{2} v_{ \pm}\left(v_{ \pm}-v_{s \pm}\right)+p_{ \pm}^{*}=p_{f \pm}^{*}
\end{aligned}
$$

Here $v_{s \pm}$ denote the shocks 3 -velocity, $v_{ \pm}$are the 3 -velocities of the fluids outside the front (i.e., ahead of the shocks) with respect to the front frame, and $\gamma_{ \pm}$are the corresponding Lorentz factors. Since we are merely interested in the strong shock limit, we shall restrict our analysis, in what follows, to the ultrarelativistic case, viz., $\gamma_{ \pm}>>1$. In this limit we can approximate the enthalpy inside the front as, $h_{f \pm} \simeq\left[\Gamma_{f \pm} /\left(\Gamma_{f \pm}-1\right)\right]\left(p_{f \pm} / r_{f \pm}\right)$. Assuming that the fluids upstream the shocks are cold (i.e., $p_{ \pm}=0$ ), eqs. (9) yield for the shock velocities,

$$
v_{s \pm}= \pm \frac{\left(2+u_{A f \pm}^{2}\right) \Gamma_{f \pm}-2}{2+\Gamma_{f \pm} u_{A f \pm}^{2}}
$$

and for the front pressure and temperature to the right (left) of the contact discontinuity, 


$$
\begin{aligned}
\frac{p_{f \pm}}{r_{ \pm} c^{2} \gamma_{ \pm}^{2}} & =\frac{4 \Gamma_{f \pm}\left(\Gamma_{f \pm}-1\right)\left(1+u_{A \pm}^{2}\right)\left(1+u_{A f \pm}^{2}\right)}{\left(2+\Gamma_{f \pm} u_{A f \pm}^{2}\right)\left(2 \Gamma_{f \pm}-2+\Gamma_{f \pm} u_{A f \pm}^{2}\right)} \\
\frac{k T_{f \pm}}{m c^{2} \gamma_{ \pm}} & \equiv \frac{p_{f \pm}}{r_{f \pm} c^{2} \gamma_{ \pm}}=\frac{2\left(\Gamma_{f \pm}-1\right)\left(1+u_{A \pm}^{2}\right)}{2+\Gamma_{f \pm} u_{A f \pm}^{2}}
\end{aligned}
$$

Note that the shocks velocities given by eq. (10) are independent of the magnetic field outside the front.

In the ideal MHD limit, the requirement that the specific magnetic field should be continuous across the shock implies that the Alfv́en 4-velocities ahead and behind the shocks are related by,

$$
\Gamma_{f \pm} u_{A f \pm}^{4}+\left[2\left(\Gamma_{f \pm}-1\right)+\left(\Gamma_{f \pm}-4\right) u_{A \pm}^{2}\right] u_{A f \pm}^{2}-2 u_{A \pm}^{2}=0,
$$

where eqs. (10) and (11) have been used in deriving eq. (12). In the weakly $\left(u_{A \pm}<<1\right)$ and strongly $\left(u_{A \pm}>>1\right)$ magnetized cases eq. (12) simplifies to $\left(u_{A f \pm} / u_{A \pm}\right)^{2}=1 /\left(\Gamma_{f \pm}-1\right)$ and $\left(u_{A f \pm} / u_{A \pm}\right)^{2}=\left(4-\Gamma_{f \pm}\right) / \Gamma_{f \pm}$, respectively. On substituting the latter results into eqs. (10) and (11) we recover the shock velocity and downstream temperature of a perfectly conducting fluid, obtained earlier by e.g., Gallant et al. (1992); $v_{s \pm}= \pm\left(\Gamma_{f \pm}-1\right), k T_{f \pm}=$ $\left(\Gamma_{f \pm}-1\right) m c^{2} \gamma_{ \pm}$, in the weakly magnetized case, and $\left(u_{s \pm} / u_{A \pm}\right)^{2}=\left(4-\Gamma_{f \pm}\right) /\left(8-4 \Gamma_{f \pm}\right)$, $k T_{f \pm}=\left[\left(2 \Gamma_{f \pm}-2\right) /\left(4-\Gamma_{f \pm}\right)\right] m c^{2} \gamma_{ \pm}$, in the highly magnetized case. We see that in the strong shock limit considered here, a perfectly conducting front is heated to relativistic temperatures for any value of the magnetic field. However, while in the case of weakly magnetized streams all of the bulk energy per particle incident into the front as measured in the front frame, $\gamma_{ \pm} m c^{2}$, is converted into random energy (the average energy per particle in a relativistic gas is $\left.k T /\left[\Gamma_{f \pm}-1\right]\right)$, in the highly magnetized case only a small fraction of the total bulk energy carried by the flow outside the front, specifically the fraction carried by the particles $\sim \gamma_{A \pm}^{-2}$ (see eq. [3]), is thermalized; the remainder of the energy is used up to compress the magnetic field inside the front. Consequently, collisions of highly magnetized flows in the ideal MHD limit lead to the formation of magnetically dominated fronts.

The situation could be markedly different if the front is highly dissipative. Then, $u_{A f \pm}$ can deviate substantially from the maximum value given by eq. (12). As seen from eq. (11), the energy per particle, $k T_{f \pm} /\left(\Gamma_{f \pm}-1\right)$, increases as $u_{A f \pm}$ decreases, ultimately approaching $m c^{2} \gamma_{ \pm} \gamma_{A \pm}^{2}$ in the limit of rapid magnetic field dissipation, i.e., $u_{A f \pm}<1$. (We have assumed that the front is adiabatic. It is conceivable that it will become radiative at temperatures lower than that derived above if the synchrotron or inverse Compton cooling time is sufficiently short. We shall not consider this possibility in this Letter.) The shock velocities approach $v_{s \pm}= \pm\left(\Gamma_{f \pm}-1\right)$ in this limit.

Now, the velocity of the contact discontinuity and, hence, the velocities upstream of each shock with respect to the front frame, $u_{ \pm}$, are unknown a priori. The requirement that the total pressure inside the front is continuous across the contact discontinuity surface yields, in the strong shock limit, the relation,

$$
\gamma_{+} \simeq \eta \gamma_{-} ; \quad \eta^{2}=\frac{r_{-} \Gamma_{f-}\left(1+u_{A-}^{2}\right)\left(1+u_{A f-}^{2}\right)\left(2+\Gamma_{f+} u_{A f+}^{2}\right)}{r_{+} \Gamma_{f+}\left(1+u_{A+}^{2}\right)\left(1+u_{A f+}^{2}\right)\left(2+\Gamma_{f-} u_{A f-}^{2}\right)} .
$$


Note that in the case of a symmetric front, which prevails when $r_{-}=r_{+}, p_{-}=p_{+}$, and $u_{A-}=u_{A+}$, eq. (13) gives $\eta=1$ as it should of course.

To complete the solution, we transform to the frame in which the fluid is injected (hereafter referred to as injection frame; this could be e.g., the rest frame of a star or a black hole from which a jet is emerged), and in which the velocities of the initial and injected flows, denoted here by $u_{+}^{\prime}$ and $u_{-}^{\prime}$, are specified. The proper mass density, pressure, and magnetic field are of course relativistic invariants and have the same values in the injection frame as in the front frame. We consider the case $u_{-}^{\prime}>>u_{+}^{\prime} \geq 0$, namely both the initial and injected fluids propagate in the same direction. On transforming to the injection frame we obtain,

$$
\gamma_{ \pm}=\gamma_{ \pm}^{\prime} \gamma_{c}-u_{ \pm}^{\prime} u_{c}
$$

with $u_{c}$ being the front 4 -velocity with respect to the injection frame. Equations (13) and (14) can be solved now to yield $\gamma_{c}$ and $\gamma_{-}$. One finds

$$
\begin{aligned}
\gamma_{c} & =\frac{\eta u_{-}^{\prime}-u_{+}^{\prime}}{\sqrt{\left(\eta u_{-}^{\prime}-u_{+}^{\prime}\right)^{2}-\left(\eta \gamma_{-}^{\prime}-\gamma_{+}^{\prime}\right)^{2}}} \\
\gamma_{-} & =\frac{\eta\left(\gamma_{-}^{\prime}-u_{-}^{\prime}\right)-\left(\gamma_{+}^{\prime}-u_{+}^{\prime}\right)}{\sqrt{\left(\eta u_{-}^{\prime}-u_{+}^{\prime}\right)^{2}-\left(\eta \gamma_{-}^{\prime}-\gamma_{+}^{\prime}\right)^{2}}}
\end{aligned}
$$

For initial fluid at rest $\left(u_{+}^{\prime}=0\right)$ the above solution simplifies to $\gamma_{-} \simeq\left(\gamma_{-}^{\prime} / 2 \eta\right)^{1 / 2}$, and for relativistic initial fluid to $\gamma_{-} \simeq\left(\gamma_{-}^{\prime} / 4 \eta \gamma_{+}^{\prime}\right)^{1 / 2}$. On substituting the latter results into eqs. (10) and (11) we obtain an expression for the front quantities in terms of $u_{ \pm}^{\prime}$. Evidently, for roughly symmetric fronts ( $\eta$ order unity) the front temperature scales roughly as $\left(\gamma_{-}^{\prime} / 4 \gamma_{+}^{\prime}\right)^{1 / 2}$. In the asymmetric case, one side of the front will be heated to much higher temperatures than the other. Heat conduction across the contact discontinuity surface may then become important if the magnetic field is not completely transverse there.

\section{DISCUSSION}

A highly conducting front produced by overtaking collisions of strongly magnetized, relativistic flows should be magnetically dominated, and is not an efficient converter of bulk energy into random energy (i.e., thermal energy or nonthermal distributions of accelerated particles). Much higher temperatures can be attained in the presence of rapid magnetic field dissipation inside the front, that may arise from magnetic reconnection or plasma instabilities near the shocks. A fraction of the dissipated field energy is likely to be radiated away in the form of electromagnetic emission with relatively high efficiency, owing to synchrotron and inverse Compton cooling of the energetic particles. The latter process can be very effective in the presence of background radiation with roughly isotropic distribution, as is believed to be the case for Galactic and extragalactic jets (Dermer \& Schlickheiser 1993; Sikora et al. 1994; Ghisellini \& Madau 1996; Levinson \& Blandford 1996). The radiative friction 
may also provide a mean for dissipating the magnetic field. Such fronts may explain the transient emission observed in many of these sources (Romanova \& Lovelace 1997). However, in order to be efficient radiator, the front must be created at not too large a distance from the putative black hole, depending on the intensity and spectrum of the external radiation, thereby imposing a constraint on the magnetosonic speed (see discussion following eq. 8). In the case of streams consisting of electron-positron plasma, the front may become loaded with $e^{ \pm}$pairs as a result of effective pair production. Frequent creation of such fronts may lead to a transition from magnetic to particle dominated jet (Levinson 1996), as seems to be suggested by observations (Blandford \& Levinson 1995). Again, the location at which the front is created might be important; the fronts must form above the cooling radius, which depends upon the intensity and spectrum of the background radiation, in order to avoid catastrophic X-ray production and the disruption of the jet.

We thank R.D. Blandford for useful comments. A.L acknowledges support from NSF grants AST 91-19475 and AST 93-15375, NASA grant NAG 5-3097, and Alon Fellowship. 


\section{REFERENCES}

[1] Begelman, M.C., \& Li, Z-Y. 1994, ApJ, 426, 269

[2] Blandford R.D., \& Eichler, D. 1987, Phys. Rep. 154, 1

[3] Blandford R.D., \& Levinson, A. 1995, ApJ, 441, 79

[4] Burns, M.L., \& Lovelace R.V.E. 1982, ApJ, 262, 87

[5] de Hoffmann F., \& Teller, E. 1958, Phys. Rev., 80, 692

[6] Dermer, C., \& Schlickheiser, R. 1993, ApJ, 416, 458

[7] Gallant, Y.A., et al. 1992, ApJ, 391, 73

[8] Ghisellini, G., \& Madau, P. 1996, MNRAS., 280, 67

[9] Kennel C.F., \& Coroniti, F.V. 1984, ApJ, 283, 694

[10] Levinson A., \& Blandford, R.D. 1996, ApJ, 456, L29

[11] Levinson, A. 1996, ApJ, 467, 546

[12] Romanova, M.M., \& Lovelace, R.V.E. 1997, ApJ, 475, 97

[13] Sikora, M., Begelman, M.C., \& Rees, M. 1994, ApJ, 421, 153

[14] Smolsky M.V., \& Usov, V.V. 1996, ApJ, 461, 858

[15] Thompson C., \& Duncan, R.C. 1993, ApJ, 408, 194

[16] Usov, V.V. 1992, Nture 357, 472

[17] van Putten, M.H.P.H. 1991, Commun. Math. Phys., 141, 67

[18] van Putten, M.H.P.H. 1993, J. Comput. Phys., 105, 339

[19] Waxman, E. 1995, Phys. Rev. Lett. 75, 386 


\section{Figure Captions}

Figure 1. Three epochs are shown in two examples of a 1D simulation of the formation of a relativistically hot, magnetized front in which the magnetic field is everywhere transverse to the fluid flow. Displayed are the temperature, the specific magnetic field, and the fluid 3 -velocity in the reference frame co-moving with the initial flow (ahead of the front). A burst of new outflow from the left is modeled by the initial 4-velocity distribution $u^{x}(x)=$ $\sinh \left\{1+\tanh \left(10 \frac{x_{0}-x}{L}\right)\right\}$, where $x$ is the coordinate along the outflow, $x_{o}$ represents the injection point and $L$ is the length of the displayed computational grid. The initial density $(r=1)$, pressure $(P=0.025)$ and Alfv́en 4-velocity $\left[u_{A}=0.5\right.$ in (a) and $u_{A}=1$ in (b)] are taken to be uniform. As seen, the higher Alfv́en velocity in (b) results in a broader front. Note the gradual steepening of the leading shock front, which is more delayed in (b). The left shock becomes steady at a very early stage in both examples shown. 
Temperature (a)

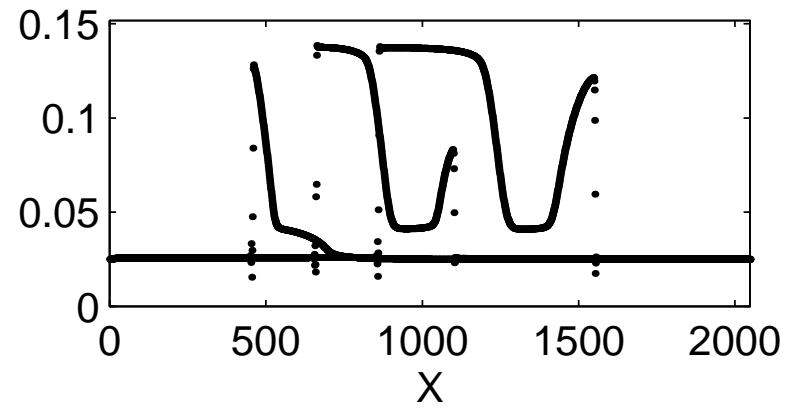

Magnetic Field (a)

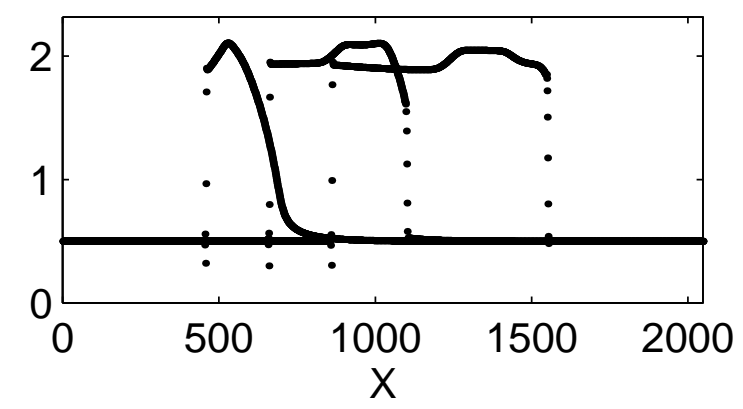

Velocity (a)

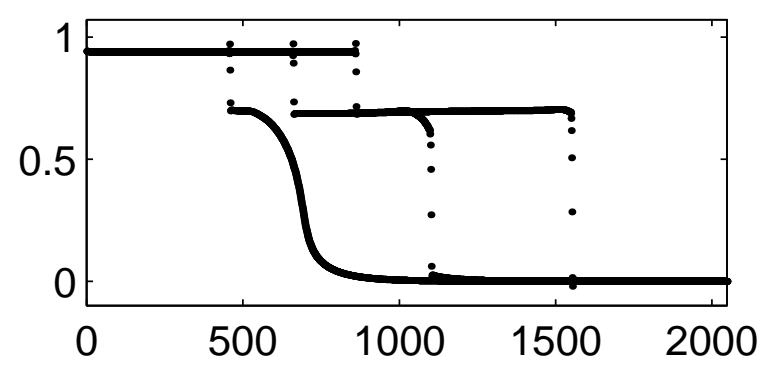

Temperature (b)

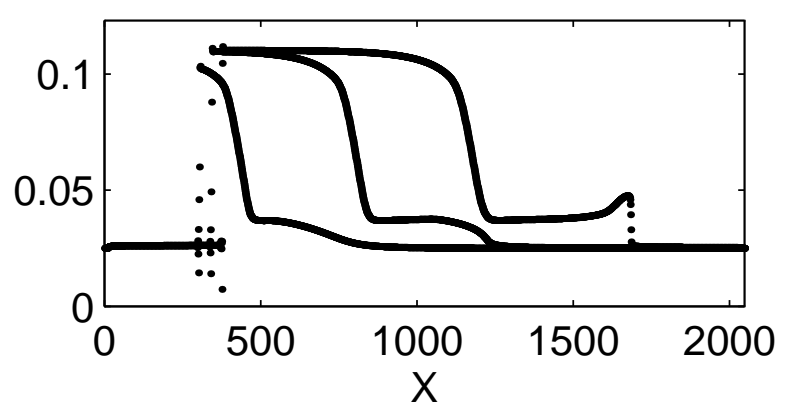

Magnetic Field (b)
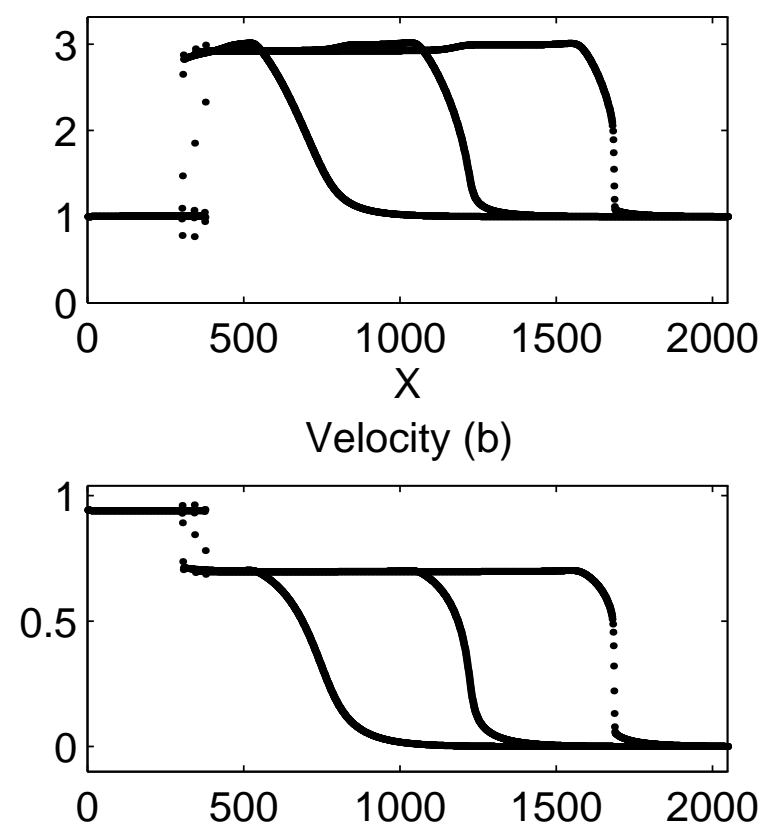